\title{
Modelo preditivo de sobrevivência do Mexilhão Dourado (Limnoperna fortunei) em relação a variações de salinidade na Laguna dos Patos, $R S$, Brasil
}

\author{
Fabiana Gonçalves Barbosa ${ }^{1,4}$ \& Adriano Sanches Melo ${ }^{2,3}$ \\ ${ }^{\text {I} P r o g r a m a ~ d e ~ P o ́ s-G r a d u a c ̧ a ̃ o ~ e m ~ E c o l o g i a, ~ D e p a r t a m e n t o ~ d e ~ E c o l o g i a, ~ I n s t i t u t o ~ d e ~ B i o c i e ̂ n c i a s, ~}$ \\ Universidade Federal do Rio Grande do Sul - UFRGS, \\ Av. Bento Gonçalves, 9500, CP 15007, CEP 91501-970, Porto Alegre, RS, Brasil \\ ${ }_{2}^{2}$ Departamento de Ecologia, Instituto de Biociências, Universidade Federal do Rio Grande do Sul - UFRGS, \\ CP 15007, CEP 91501-970, Porto Alegre, RS, Brasil \\ ${ }^{3}$ Departamento de Ecologia, Instituto de Ciências Biológicas, Universidade Federal de Goiás - UFG, \\ CP 131, CEP 74001-970, Goiânia, GO, Brasil, e-mail: asm.adrimelo@ gmail.com \\ ${ }^{4}$ Autor para correspondência: Fabiana Gonçalves Barbosa, e-mail: fabibarbos@gmail.com
}

BARBOSA, F.G. \& MELO, A.S. Predictive model of survival of the Golden Mussel (Limnoperna fortunei) in relation to variations of salinity in the Laguna dos Patos, RS, Brazil. Biota Neotrop. 9(3): http://www. biotaneotropica.org.br/v9n3/pt/abstract?short-communication+bn02809032009.

\begin{abstract}
Limnoperna fortunei (golden mussel), a freshwater bivalve native to Southeast Asia, invaded southern South America around 1991. Using species' occurrence records and salinity in different seasons in Laguna dos Patos, RS, Brazil, we predict survival probabilities in different regions of the lagoon during the different seasons of the year. We fitted the data to a logistic regression model. A total of 26 presence records (live, dead) of L. fortunei and salinity at those locations were obtained from the literature for the Laguna dos Patos in the period 2001-2004. Additional data on salinity were used to interpolate salinity values to all regions of the lagoon. The logistic regression model shows that survival decreases as salinity increases. The model predicted high survival probability of the species during periods of low salinity (austral winter) in the limnic, pre-limnic and estuarine regions of the lagoon. During the high salinity period (summer), the species is able to survive only in the limnic and pre-limnic region of the lagoon. These data suggest that control measures should be carried out most effectively during the summer.
\end{abstract}

Keywords: exotic species, invasive mussel, biological invasion, logistic regression.

BARBOSA, F.G. \& MELO, A.S. Modelo preditivo de sobrevivência do Mexilhão Dourado (Limnoperna fortunei) em relação a variações de salinidade na Laguna dos Patos, RS, Brasil. Biota Neotrop. 9(3): http:// www.biotaneotropica.org.br/v9n3/en/abstract?short-communication+bn02809032009.

Resumo: Limnoperna fortunei (mexilhão dourado), um bivalve de água doce do sudeste da Ásia, invadiu o sul da América do Sul em torno de 1991. Dados de ocorrência da espécie e salinidade ao longo do ano foram usados para predizer o potencial de sobrevivência de L. fortunei na Laguna dos Patos, RS. Um modelo preditivo foi construído usando regressão logística. Obtivemos na literatura 26 registros de presença (vivo, morto) de L. fortunei e valores de salinidade medidos em cada um destes registros na Laguna dos Patos entre o período de 2001 a 2004. Registros adicionais de salinidade foram utilizados para interpolar os valores por toda a Laguna dos Patos. Sobrevivência de L. fortunei diminui quando a salinidade aumenta na Laguna dos Patos. Os mapas de distribuição predita das áreas favoráveis à sobrevivência de L. fortunei indicam que durante os períodos de baixas salinidades as regiões límnica, pré-límnica e estuarina da laguna mostraram-se favoráveis para a sobrevivência da espécie. Nos períodos de altas salinidades (verão) a sobrevivência do molusco é favorável apenas na região límnica e na região prélímnica. Estes resultados sugerem que o controle mais efetivo desta espécie deve ser feito durante o verão.

Palavras-chave: espécies exóticas, mexilhão invasor, invasões biológicas, regressão logística. 


\section{Introdução}

As invasões biológicas constituem um grave problema ecológico em ambientes aquáticos. Tais invasões geralmente afetam a estrutura das comunidades e o próprio funcionamento do ecossistema (Williamson \& Fitter 1996). Espécies invasoras geralmente são capazes de se reproduzir de forma rápida, apresentam crescimento acelerado e elevada adaptação aos diferentes ambientes aquáticos que colonizam, sejam naturais ou artificiais (Darrigran 1997).

Em ambientes de água doce, os moluscos bivalves se destacam pelo grande número de espécies com elevado potencial de invasão. Diversas invasões por espécies de moluscos bivalves de água doce têm sido relatadas mundialmente, merecendo destaque as invasões de Dreissena polymorpha (Pallas, 1771) (área nativa: Europa e Ásia; área invadida: Grandes Lagos, América do Norte), Dreissena bugensis (Andrusov, 1857) (área nativa: Ucrânia; área invadida: Grandes Lagos, América do Norte), Corbicula fluminea (Muller, 1774) (área nativa: Sudeste da Ásia; área invadida: América do Norte, América do Sul, África e Europa), Corbicula fluminalis (Muller, 1774) (área nativa: Ásia; área invadida: Norte da América e Europa) e Limnoperna fortunei (Dunker, 1857) (área nativa: Sudeste da Ásia; área invadida: Hong Kong, Japão, Taiwan e América do Sul) (Karatayev et al. 2007).

Limnoperna fortunei, conhecido popularmente como mexilhão dourado, é um molusco bivalve originário da epifauna do sudeste da Ásia (Camboja, China, Coréia, Indonésia, Laos, Tailândia e Vietnã). Nos últimos 30 anos a espécie vem expandindo sua distribuição em vários ambientes aquáticos do mundo: Hong Kong, Japão, Taiwan e países da América do Sul (Argentina, Bolívia, Brasil, Paraguai e Uruguai) (Ricciardi 1998, Brugnoli et al. 2005).

O primeiro registro de ocorrência de L. fortunei na região Neotropical foi em 1991 na Bacia do Rio da Prata, Balneário de Bagliardi (34 55' S e 57 59' O), próximo de Buenos Aires, Argentina (Pastorino et al. 1993). Provavelmente, a introdução foi feita através do transporte de larvas por água de lastro de navios mercantes provenientes da Ásia. De fato, a época do aparecimento da espécie na Argentina coincidiu com os picos mais altos de importação de produtos asiáticos (Darrigran \& Pastorino 1995).

No Brasil, L. fortunei encontra-se presente no Lago Guaíba (Mansur et al. 1999), no sistema da Laguna dos Patos (Mansur et al. 2003, Capítoli \& Bemvenuti 2004), na bacia dos rios Paraguai e Paraná e ecossistemas aquáticos da região do Pantanal (Oliveira et al. 2006) e dos Estados de Minas Gerais e São Paulo (Avelar et al. 2004, Sylvester et al. 2007). Na Laguna dos Patos, a primeira ocorrência da espécie foi registrada em seu extremo setentrional (30 S) em 1998. Após dois anos desta colonização inicial, a espécie se dispersou para as regiões límnica, Arambaré (3050'S), e pré-límnica, São Lourenço do Sul (31²0’ S) (Mansur et al. 2003) (Figura 1). A partir de 2002 a espécie espalhou-se em diferentes ambientes da região sul da Laguna, como a região estuarina, o Rio Pelotas e a porção estuarina do Canal São Gonçalo (Capítoli \& Bemvenuti, 2004, 2005, Burns et al. 2006, Piedras et al. 2007, Capítoli et al. 2008).

Pesquisas de laboratório sobre a influência da variação de salinidade na sobrevivência de L. fortunei foram desenvolvidas por Deaton et al. (1989) e Angonesi et al. (2008). Além destas pesquisas, estudos em ambientes costeiros relacionados à presença e distribuição de L. fortunei relatam que, entre uma série de variáveis ambientais (temperatura, oxigênio dissolvido, $\mathrm{pH}$ ), a salinidade pode ser considerada um fator limitante à disseminação da espécie (Darrigran 1995, Darrigran \& Pastorino 1995, Ricciardi 1998, Darrigran 2002, Capítoli \& Bemvenuti 2004, 2005, Brugnoli et al. 2005, Piedras et al. 2007).

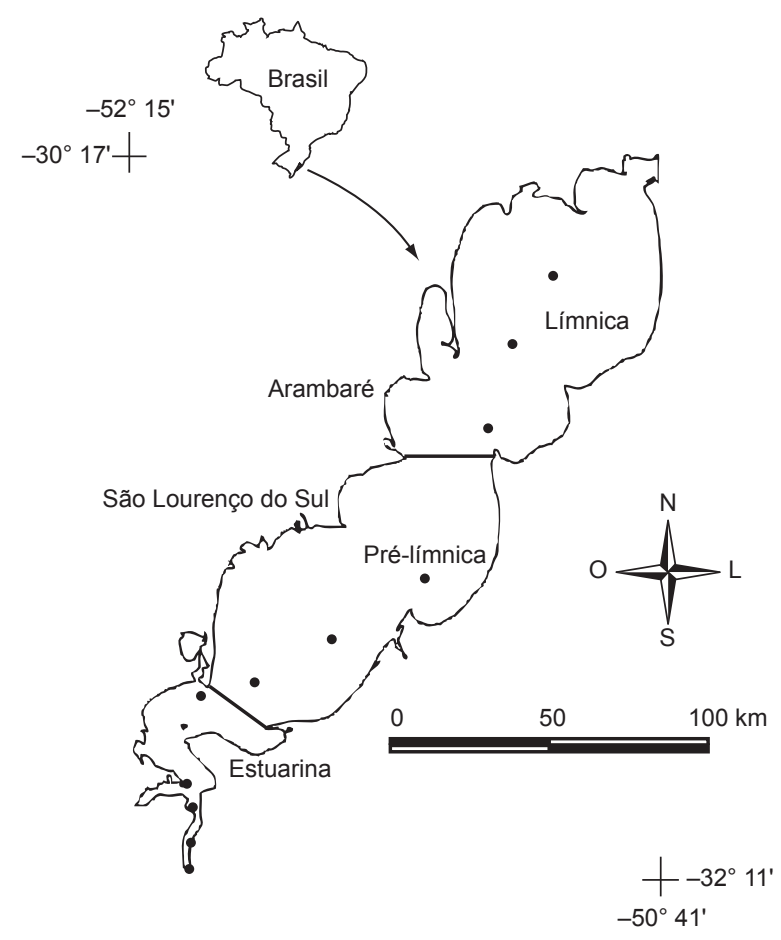

Figura 1. Laguna dos Patos e sua divisão ecológica. Os 11 pontos marcados representam os locais onde foram obtidos os valores de salinidade entre 2000-2004.

Figure 1. Laguna dos Patos and its ecological division. The 11 dots indicate the locations where data of salinity between 2000-2004 were obtained.

Na região estuarina da Laguna dos Patos o aporte de águas salgadas provenientes do Oceano Atlântico é essencialmente regido pelas condições meteorológicas da região, principalmente pelo regime de precipitação pluvial e intensidade dos ventos (Möller et al. 2001, Fernandes et al. 2004). De acordo com as diferentes combinações entre a intensidade deste aporte e a direção do vento incidente na Laguna dos Patos, a salinidade da laguna tende a variar ao longo do seu comprimento, experimentando variações que alcançam de 0-34 ups (unidade padrão de salinidade) (Castello 1985).

Por essa razão, espera-se identificar faixas de salinidade que possam limitar a distribuição e persistência de L. fortunei na Laguna dos Patos. Além disso, tais informações poderão fornecer subsídios para futuras ações preventivas na região, pois segundo Oliveira et al. (2006), uma vez presente no ambiente a erradicação da espécie é extremamente difícil.

Neste trabalho compilamos dados da literatura quanto à presença de L. fortunei em diferentes locais da Laguna dos Patos e relacionamos a condição dos indivíduos (vivos ou mortos) à salinidade. Especificamente, nossos objetivos foram determinar o nível de salinidade tolerado pela espécie em ambiente natural e produzir mapas de sobrevivência potencial nas quatro estações do ano.

\section{Material e Métodos}

\section{1. Área de estudo}

A Laguna dos Patos, situada na planície costeira do Estado do Rio Grande do Sul, Brasil, representa a maior área lagunar da América do Sul e a maior laguna costeira do tipo "estrangulada" do mundo (Kjerfve 1986). Esta laguna estende-se ao longo de $250 \mathrm{~km}$ de comprimento, possuindo uma área superficial de $10.360 \mathrm{~km}^{2} \mathrm{e}$ 
drena uma bacia hidrográfica de aproximadamente $200.000 \mathrm{~km}^{2}$ (Möller et al. 1996).

O sistema Laguna dos Patos destaca-se entre as regiões costeiras do Atlântico Sudoeste pela sua importância ecológica (zona de produção biológica e diversidade) (Asmus \& Tagliani 1998). A laguna pode ser dividida em três regiões ecológicas distintas: i) a região límnica, que compreende a célula do setor norte da Laguna dos Patos; ii) a região pré-límnica, que se estende desde $31^{\circ} 05^{\prime} \mathrm{S}$ até o limite da região estuarina; e iii) a região estuarina, na porção sul da laguna, delimitada ao norte por uma linha imaginária traçada entre a Ponta da Feitoria $\left(31^{\circ} 41^{\prime} \mathrm{S}\right.$ e $\left.52^{\circ} 02^{\prime} \mathrm{O}\right)$ à Ponta dos Lençóis $\left(31^{\circ} 48^{\prime} \mathrm{S}\right.$ e $\left.51^{\circ} 50^{\prime} \mathrm{O}\right)$ e ao sul pelos molhes da Barra ( $32^{\circ} 11^{\prime} \mathrm{S}$ e $\left.52^{\circ} 04^{\prime} \mathrm{O}\right)$ (Capítoli \& Bemvenuti 2004) (Figura 1).

\section{Dados bióticos e abióticos}

Compilamos da literatura um total de 26 registros de presença (vivo ou morto) de L. fortunei e valores de salinidade medidos para cada um destes registros na Laguna dos Patos entre o período de 2001 a 2004 (Capítoli \& Bemvenuti 2004, 2005, Piedras et al. 2007). Além destes dados, também compilamos dados georreferenciados de salinidade em 11 localidades de estudos realizados na Laguna dos Patos durante o mesmo período (Pereira 2003, Barbosa 2006) (Figura 1).

\section{Análise estatística dos dados}

Usamos um modelo de regressão logística simples para predizer o potencial de sobrevivência de L. fortunei na Laguna dos Patos sob diferentes condições de salinidade. O modelo de regressão logística resulta numa equação preditiva da ocorrência da variável resposta em função de uma variável preditora. Especificamente, o modelo produz valores de probabilidade de que uma variável binária assuma um certo valor, em função da variável preditora contínua (Keating \& Cherry 2004).

Neste estudo, a variável resposta binária é a condição de L. fortunei (vivo ou morto) em localidades na Laguna dos Patos e a variável preditora é a salinidade mensurada em cada uma destas localidades. O modelo de regressão ajustado aos dados observados foi então usado para estimar a probabilidade de sobrevivência da espécie em toda a área da laguna. Valores de probabilidade próximos de 0 indicam que o valor de salinidade presente em determinado local da laguna não é favorável para a sobrevivência de L. fortunei, enquanto valores próximos de 1 indicam que existe uma forte probabilidade de sobrevivência da espécie naquele local em função da existência de um valor de salinidade favorável. Para a análise de regressão logística utilizamos o ambiente de programação R, versão 2.4.1 (The R Development Core Team 2006)

\section{Estimativa de valores de salinidade}

Para extrapolação dos resultados para toda a Laguna dos Patos, utilizamos valores de salinidade independentes daqueles utilizados na construção do modelo de regressão logística. A não inclusão das informações de salinidade associadas aos registros de $L$. fortunei devese ao fato de parte destes terem sido obtidos em somente uma estação climática do ano e, principalmente, pela inexistência de coordenadas geográficas destas localidades. Assim, usamos um conjunto de dados de salinidade obtido em 11 localidades, na superfície da água, dispostas ao longo do eixo maior da Laguna dos Patos (Figura 1). Os valores de salinidade foram obtidos entre janeiro de 2000 e março de 2004, geralmente com amostragem mensal. Na análise, agrupamos os dados de acordo com a estação do ano e obtivemos médias para cada uma das localidades nas quatro estações climáticas.
Utilizamos os valores de salinidade nas 11 localidades para interpolar valores em 15 outras localidades no eixo principal da Laguna dos Patos, distantes $10 \mathrm{~km}$ entre si. A estimativa de salinidade para cada estação climática nas 15 localidades foi feita de acordo com a Equação 1 abaixo:

$$
\mathrm{S}_{\mathrm{B}}=\left(\mathrm{S}_{\mathrm{C}}-\mathrm{S}_{\mathrm{A}}\right) \times\left(\mathrm{d}_{\mathrm{AB}} / \mathrm{d}_{\mathrm{AC}}\right)+\mathrm{S}_{\mathrm{A}}
$$

onde, $\mathrm{S}_{\mathrm{B}}=$ valor estimado de salinidade na localidade $\mathrm{B}, \mathrm{S}_{\mathrm{A}}$ e $S_{C}=$ valores de salinidade observados nas localidades adjacentes A e C, $d_{A B}$ e $d_{A C}=$ distâncias entre as localidades A-B e A-C. Esses valores estimados juntamente com aqueles observados serviram de base para elaborarmos um MNT (Modelo Numérico de Terreno) de salinidade para cada estação climática. O MNT é utilizado para denotar a representação de uma grandeza que varia continuamente no espaço e que compreende i) a amostragem para aquisição de dados reais; e ii) a geração de grades que permitem obter uma melhor visualização e espacialização dos dados a partir das amostras (Câmara \& Medeiros 2005). O MNT foi feito com resolução de $10 \mathrm{~km}$ no eixo latitudinal. Assumimos que os valores de salinidade não variam longitudinalmente de forma significativa. Utilizamos o interpolador Média Ponderada por Quadrante para geração de grades usando o programa SPRING versão 4.3.3 (INPE 2007). Extraímos a base cartográfica da Laguna dos Patos, na escala 1:250.000, da base de dados disponível em FEPAM (2005).

A partir dos valores de salinidade, incluídos nos MNTs, executamos o fatiamento para prever a condição de sobrevivência de L. fortunei em toda a Laguna dos Patos segundo o modelo de regressão logística obtido. Para o fatiamento, usamos os resultados obtidos na regressão logística e classificamos a sobrevivência em: i) área muito favorável (sobrevivência maior que 75\%); ii) área relativamente favorável (sobrevivência entre 50 e 75\%); e iii) área desfavorável à sobrevivência de L. fortunei (sobrevivência menor que 50\%).

\section{Resultados}

A sobrevivência de L. fortunei na Laguna dos Patos foi afetada negativamente pela salinidade $(\mathrm{z}=-2,59 ; \mathrm{p}=0,009)$. A sobrevivência foi elevada em águas com salinidade de até 5 ups, mas decresceu rapidamente em salinidades mais altas (Figura 2).

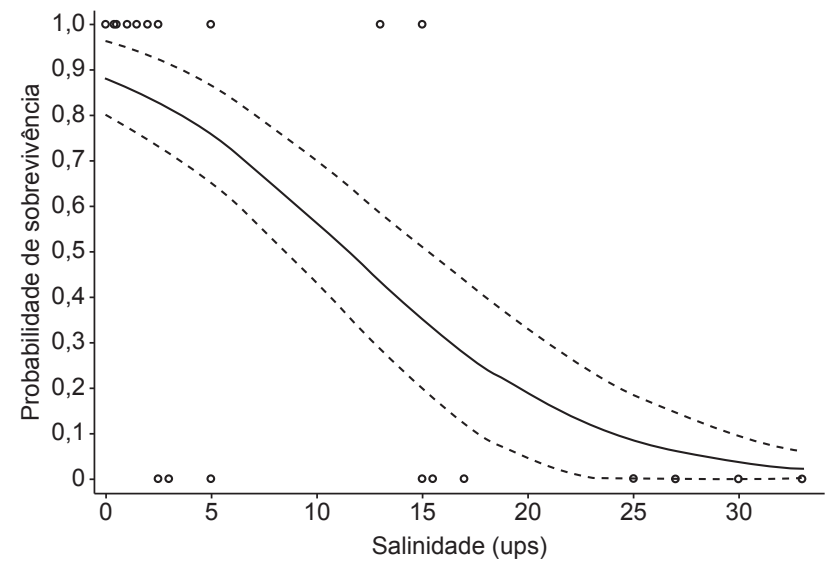

Figura 2. Dados observados e modelo de regressão logística ajustado aos dados de sobrevivência de Limnoperna fortunei em relação à salinidade na Laguna dos Patos. As linhas pontilhadas indicam o erro padrão. $\mathrm{y}=\mathrm{e}^{(2,008-0,174 * \mathrm{X})}$ $/\left(1+\mathrm{e}^{\left(2,008-0,174^{*} \mathrm{X}\right)}\right)$.

Figure 2. Observed data and logistic regression model adjusted to the data of survival of the Limnoperna fortunei in relation to salinity in Laguna dos Patos. Dotted lines indicate the standard error. $\mathrm{y}=\mathrm{e}^{(2.008-0.174 * \mathrm{X})} /\left(1+\mathrm{e}^{(2.008-0.174 * \mathrm{X})}\right)$. 

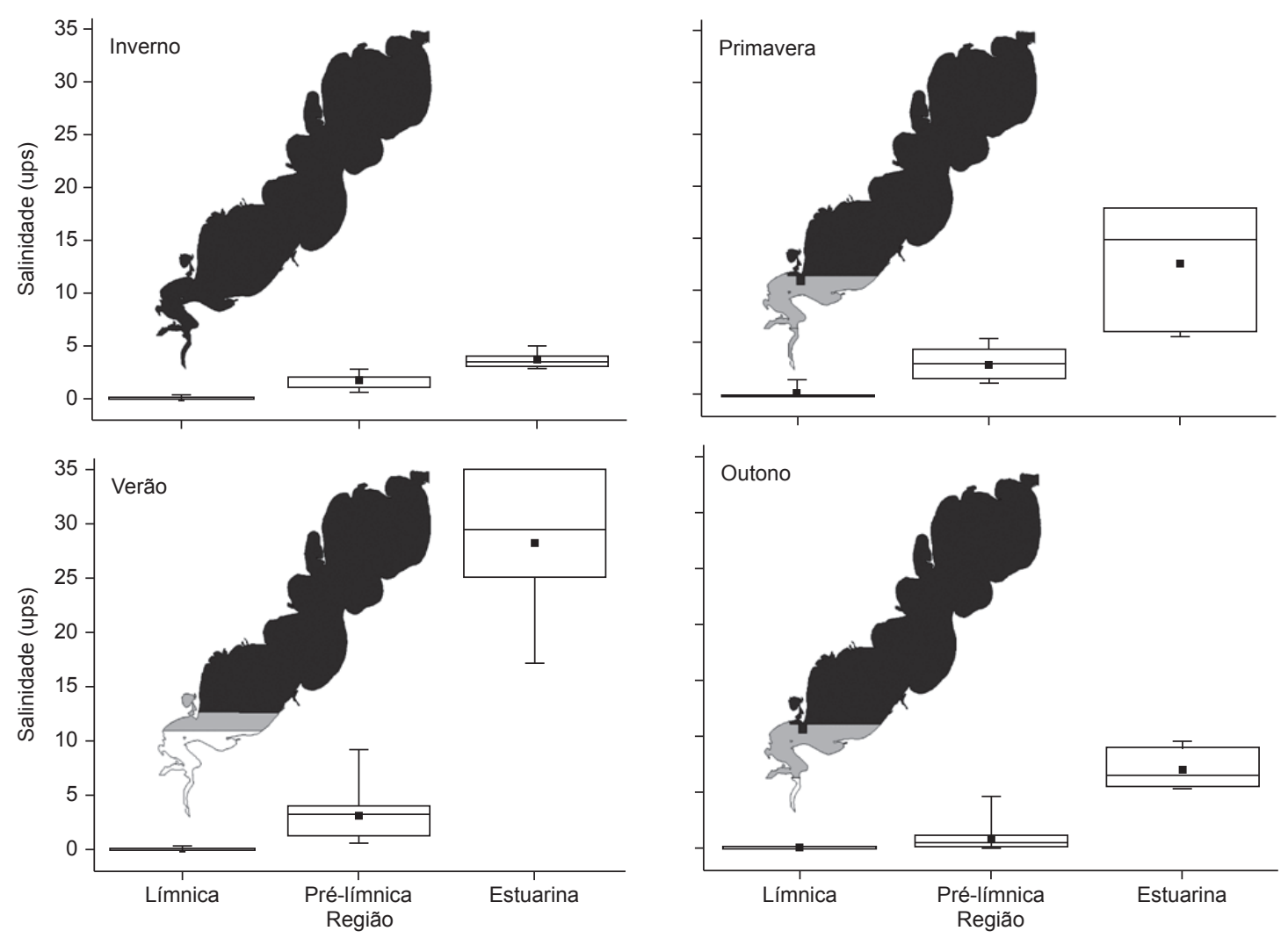

Figura 3. Mapas de distribuição predita das áreas favoráveis à sobrevivência de Limnoperna fortunei na Laguna dos Patos (Rio Grande do Sul, Brasil) em função da salinidade para cada estação climática. Salinidade nas regiões ecológicas da Laguna dos Patos para as estações climáticas registradas de 2000 a 2004. As caixas representam os percentis 25 e $75 \%$. A linha horizontal dentro da caixa representa a mediana e o ponto a média. As barras de erro (whiskers) representam o mínimo e o máximo.

Figure 3. Predicted distribution maps of the areas favorable for survival of the Limnoperna fortunei in Laguna dos Patos (Rio Grande do Sul, Brazil) as a function of salinity for each climate station. Salinity in the ecological regions of the Laguna dos Patos for climate stations recorded from 2000 to 2004 . The boxes represent the percentiles 25 and $75 \%$. The horizontal line within the box represents the median and the dot indicate the mean. The error bars (whiskers) represent the minimum and maximum.

As maiores salinidades ocorreram nas estações de verão, outono e primavera e as menores salinidades no inverno (Figura 3). De acordo com o modelo de regressão logística ajustado aos 26 registros de salinidade observados, podemos classificar a sobrevivência de $L$. fortunei na Laguna dos Patos da seguinte maneira: i) área muito favorável - sobrevivência $>75 \%(0<$ salinidade $<5,1)$; ii $)$ área relativamente favorável $-75 \%>$ sobrevivência $>50 \%(5,1<$ salinidade $<11,6)$; e iii) área desfavorável - 50\% > sobrevivência $(11,6<$ salinidade $<33)$ (Figura 3).

Durante o período de baixas salinidades (inverno), as regiões límnica, pré-límnica e estuarina da Laguna dos Patos mostraram-se favoráveis à sobrevivência da espécie (acima de 75\%). No período de altas salinidades (verão), a região estuarina apresentou-se desfavorável à sobrevivência do molusco (Figura 3).

\section{Discussão}

A previsão de sobrevivência de L. fortunei na Laguna dos Patos concentrou-se nas regiões límnica e pré-límnica, independente das estações climáticas. Esta espécie foi registrada no ano de 2000 na região límnica (Arambaré) e em 2001 na região pré-límnica (São Lourenço do Sul) (Mansur et al. 2003) e atualmente as populações que colonizaram essas regiões podem ser consideradas persistentes.
Na região estuarina, o modelo indicou essa região como inóspita à espécie em altas salinidades (verão). As grandes variações de salinidade na área (0-33 ups) impedem o estabelecimento da espécie. Estes resultados são concordantes com os de Capítoli \& Bemvenuti (2004, 2005) e de Capítoli et al. (2008) que registraram a mortalidade de populações da espécie que se desenvolveram no corpo do estuário durante a penetração da cunha salina pelo Canal do Rio Grande.

De modo geral, a predição das áreas potencialmente favoráveis à sobrevivência de L. fortunei na Laguna dos Patos situou-se dentro dos padrões descritos por diversos autores para outras zonas costeiras (Darrigran 1995, Darrigran \& Pastorino 1995, Ricciardi 1998, Brugnoli et al. 2005). Apesar de ser um molusco bivalve de água doce, L. fortunei tolera concentrações salinas (Deaton et al. 1989) que vão desde 0 a 12 ups e mantém grandes populações em regiões estuarinas (Ricciardi 1998, Darrigran 2002).

No estuário do Rio da Prata, Argentina, Darrigran \& Pastorino (1995) analisaram o modelo de distribuição de L. fortunei e constataram que a distribuição da espécie é limitada por altos valores de salinidade e que a mesma resiste a salinidades de até 3 ups. Em outro estudo, na costa Uruguaia do Rio da Prata, o papel da salinidade como o principal fator abiótico limitante da distribuição de L. fortunei foi descrito por Brugnoli et al. (2005). Segundo estes autores, L. fortunei ocorre nas áreas correspondentes às isohalinas de valores 2-3 ups 
em Punta Espinillo e nas localidades de Colônia e Montevidéu com salinidades de 0 e 1,6 ups respectivamente.

Estudos recentes sobre a distribuição de L. fortunei em distintos ambientes da Laguna dos Patos (Burns et al. 2006, Piedras et al. 2007) sugerem que as colonizações e/ou persistências ocorrem concomitantemente à predominância de baixas salinidades. A salinização é um fator natural controlador da disseminação de $L$. fortunei durante um período de salinização do baixo Arroio Pelotas, localizado no município de Pelotas (Piedras et al. 2007). Entretanto, a espécie manteve estoques populacionais persistentes em locais com salinidade inferior a 5 ups. No Canal de São Gonçalo, que conecta o estuário da Laguna dos Patos com a Laguna Mirim, foram encontradas populações estabelecidas de L. fortunei na região límnica, enquanto que na região estuarina deste canal as populações não conseguem se estabelecer devido aos eventos de estiagem e salinização (Burns et al. 2006).

Além de observações na natureza, pesquisas de laboratório avaliaram a influência da variação da salinidade na sobrevivência de L. fortunei. Entre estas, podem ser destacadas as realizadas tanto no continente asiático (Deaton et al. 1989) como na América do Sul (Angonesi et al. 2008). Esta espécie é capaz de suportar valores menores do que 3 ups, $80 \%$ dos exemplares expostos a salinidade 7 ups morrem em 3 semanas e $84 \%$ dos expostos a 11 ups morrem em uma semana (Deaton et al. 1989). O mexilhão dourado pode sobreviver (90\%) em condições de choque quando submetidos a águas com salinidade 2 ups por períodos de no mínimo 10 dias. Em testes de choque com salinidade 4 e 6 ups esta espécie mostrou $20 \%$ de sobrevivência por período de 10 dias (Angonesi et al. 2008).

Nossos mapas preditivos apresentaram uma distribuição de sobrevivência de L. fortunei perante variações de salinidade na Laguna dos Patos bastante precisa, especialmente em comparação aos registros históricos da distribuição do mexilhão, os quais mostram que a salinidade pode ser considerada uma variável aquática limitante na sobrevivência de L. fortunei na região estuarina da Laguna dos Patos. Neste contexto, nosso mapa preditivo para estação climática de verão pode fornecer subsídios para ações de controle à distribuição e persistência de L. fortunei na região. Adicionalmente, sugerimos que trabalhos futuros, em áreas com variações significativas de salinidade, utilizem esta variável na modelagem da distribuição da espécie.

\section{Agradecimentos}

Nossos agradecimentos à Eliana Lima da Fonseca, Fernando Gertum Becker e dois revisores anônimos pelas importantes sugestões ao manuscrito. Agradecemos também à Coordenação de Aperfeiçoamento de Pessoal de Nível Superior (CAPES) pela concessão de Bolsa de Doutorado à primeira autora. Este projeto contou com recursos de projetos financiados pelo Conselho Nacional de Desenvolvimento Científico e Tecnológico (CNPq 476256/2004-6, 476304/2007-5) e International Foundation for Science (IFS no. A/4107-1).

\section{Referências Bibliográficas}

ANGONESI, L.G., ROSA, N.G. \& BEMVENUTI, C.E. 2008. Tolerance to salinities shocks of the invasive mussel Limnoperna fortunei under experimental conditions. Iheringia Ser. Zool. 98(1):66-69.

ASMUS, M.L. \& TAGLIANI, P.R.A. 1998. Considerações sobre o manejo ambiental. In Os ecossistemas costeiro e marinho do extremo Sul do Brasil (U. Seeliger, C. Odebrecht \& P. Castello, eds). Ecoscientia, Rio Grande, p. 225-229.

AVELAR, W.E.P., MARTIM, S.L. \& VIANNA, M.P. 2004. A new occurrence of Limnoperna fortunei (Dunker, 1857) (Bivalvia, Mytilidae) in the state of São Paulo, Brazil. Rev. Bras. Biol. 64(4):739-742.

BARBOSA, F.G. 2006. Variações espaciais e temporais de nutrientes dissolvidos e metais traço na área portuária da cidade do Rio Grande
(Estuário da Lagoa dos Patos - RS). Dissertação de Mestrado, Fundação Universidade Federal do Rio Grande, Rio Grande, 152p.

BRUGNOLI, E., CLEMENTE, J., BOCCARDI, L., BORTHAGARAY, A. \& SCARABINO, F. 2005. Golden mussel Limnoperna fortunei (Bivalvia: Mytilidae) distribution in the main hydrographical basins of Uruguay: update and predictions. An. Acad. Bras. Cienc. 77(2):235-244.

BURNS, M.D.M., GERALDI, R.M., GARCIA, A.M., BEMVENUTI, C.E., CAPITOLI, R.R. \& VIEIRA, J.P. 2006. Primeiro registro de ocorrência do mexilhão dourado Limnoperna fortunei na bacia de drenagem da Lagoa dos Patos, RS, Brasil. Biociências. 14(1):83-84.

CÂMARA, G. \& MEDEIROS, J.S. 2005. Modelagem de dados em geoprocessamento. In Sistemas de Informação Geográfica: aplicações na agricultura (E.D. Assad \& E.E. Sano, eds). EMBRAPA, Brasília, p. 47-66.

CAPÍTOLI, R.R. \& BEMVENUTI, C.E. 2004. Distribuição do mexilhão dourado Limnoperna fortunei (Dunker, 1857) na área estuarina da Lagoa dos Patos e Canal São Gonçalo. In Anais do VI Simpósio de Ecossistemas Brasileiros. Academia de Ciências do Estado de São Paulo. 110(1):98-107.

CAPÍTOLI, R.R. \& BEMVENUTI, C.E. 2005. Cenários de distribuição e processo de colonização do mexilhão dourado Limnoperna fortunei (Dunker, 1857) entre as bacias da Lagoa dos Patos e Lagoa Mirim (RS - BRASIL). In Anais do I Simpósio Brasileiro sobre Espécies Exóticas Invasoras. Brasília, p. 1-17. http://www.mma.gov.br/sitio/index. php?ido=conteudo.monta\&idEstrutura=174\&idConteudo=7966. (último acesso em 25/07/2009).

CAPÍTOLI, R.R., COLLING, L.A. \& BEMVENUTI, C.E. 2008. Cenários de distribuição do mexilhão dourado Limnoperna fortunei (Mollusca Bivalvia) sob distintas condições de salinidade no complexo Lagunar Patos-Mirim, RS - Brasil. Atlântica. 30(1):35-44.

CASTELlO, J.P. 1985. La ecologia de los consumidores del estuário de la Lagoa dos Patos, Brasil. In Fish community ecology in estuaries and coastal lagoons: towards an ecossystem integration (A. YañezArancibia, ed.). Universidade Autônoma Nacional do México, México, p. 386-406.

DARRIGRAN, G. 1995. Limnoperna fortunei: um problema para los sistemas naturales de água dulce del Mercosur? Revista Museo La Plata. 1(5):85-87.

DARRIGRAN, G. 1997. Invading bivalves in South America. Aliens. 5:3-4.

DARRIGRAN, G. 2002. Potential impact of filter-feeding invaders on temperate inland fresh water environments. Biol. Invasions. 4(1-2):145-156.

DARRIGRAN, G. \& PASTORINO, G. 1995. The recent introduction of a freshwater asiatic bivalve Limnoperna fortunei (Mytilidae) into South America. Veliger. 32(2):171-175.

DEATON, L.E., DERBY, J.S., DUHEDAR, N. \& GREENBERG, M.J. 1989. Osmoregulation and salinity tolerances in two species of bivalve mollusks: Limnoperna fortunei and Mytilopsis leocophaeta. J. Exp. Mar. Biol. Ecol. 133(1-2):67-79.

FERNANDES, E.H.L., DYER, K.R. \& MÖLLER, O.O. 2004. Spatial gradients in the flow of southern Patos Lagoon. J. Coast. Res. 21(4):759-769.

Fundação Estadual de Proteção Ambiental Henrique Luiz Roessler - FEPAM. 2005. Base cartográfica digital do Rio Grande do Sul, 1:250.000, lagos e lagoas. http://www.fepam.rs.gov.br/biblioteca/geo/bases_geo.asp. (último acesso em 18/10/2007).

Instituto Nacional de Pesquisas Espaciais - INPE. 2007. Sistema de Processamento de Informações Georeferenciadas - SPRING, versão 4.3.3. http://www.dpi.inpe.br/spring (último acesso em 05/04/2008).

KARATAYEV, A.Y., PADILLA, D.K., MINCHIN, D., BOLTOVSKOY, D. \& BURLAKOVA, L.E. 2007. Changes in global economics and trade: the potential spread of exotic freshwater bivalves. Biol. Invasions. 9(2):161-180.

KEATING, K.A. \& CHERRY, S. 2004. Use and interpretation of logistic regression in habitat selection studies. J. Wildl. Manage. 68(4):774-789. 
KJERFVE, B. 1986. Comparative oceanography of coastal Lagoons. In Estuarine variability (D.A. Wolf, ed.). Academic Press, Orlando, p. 63-81.

MANSUR, M.C.D., RICHINITTI, L.M.Z. \& SANTOS, C.P. 1999. Limnoperna fortunei (Dunker, 1857) molusco bivalve invasor na bacia do Guaíba, Rio Grande do Sul, Brasil. Biociências. 7(2): 147-149.

MANSUR, M.C.D., SANTOS, C.P., DARRIGRAN, G., HEYDRICH, I., CALLIL, C.T. \& CARDOSO, F.R. 2003. Primeiros dados quali/ quantitativos do mexilhão-dourado, Limnoperna fortunei (Dunker), no Delta do Jacuí, no Lago Guaíba e na Laguna dos Patos, Rio Grande do Sul, Brasil e alguns aspectos de sua invasão no novo ambiente. Rev. Bras. Zool. 20(1):75-84.

MÖLLER, O.O., LORENZZENTTI, J.A., STECH, J.L. \& MATA, M.M. 1996. The Patos Lagoon summertime circulation and dynamics. Cont. Shelf Res. 16(3):335-351.

MÖLLER, O.O., CASTING, P., SALOMON, J.C. \& LAZURE, P. 2001. The influence of local and non local forcing effects on the subtidal circulation of Patos Lagoon. Estuaries. 24(2):297-311.

OLIVEIRA, M.D., TAKEDA, A.M., BARROS, L.F., BARBOSA, D.S. \& RESENDE, E.K. 2006. Invasion by Limnoperna fortunei (Dunker, 1857) (Bivalvia, Mytilidae) of the Pantanal wetland, Brazil. Biol. Invasions. 8(1):97-104.

PASTORINO, G., DARRIGRAN, G., MARTIN, S. \& LUNASCHI, L. 1993. Limnoperna fortunei (Dunker, 1857) (Mytilidae), nuevo bivalvo invasor en aguas del rio de La Plata. Neotropica. 39(101-102):34.
PEREIRA, R.S. 2003. Processos que regem a qualidade da água da Lagoa dos Patos, segundo o Modelo DELFT 3D. Dissertação de Mestrado, Universidade Federal do Rio Grande, Rio Grande, 138p.

PIEDRAS, S.R., BAGER, A. \& CORRÊA, F. 2007. Ocorrência de Limnoperna fortunei (Dunker, 1857) (Mytilidae) durante um período de salinização do baixo Arroio Pelotas, Pelotas, Rio Grande do Sul. Bol. Inst. Pesca. 33(1):121-125.

RICCIARDI, A. 1998. Global range expansion of the mussel Limnoperna fortunei (Mytilidae): another fouling threat to freshwater systems. Biofouling. 13(2):97-106.

SYLVESTER, F., BOLTOVSKOY, D. \& CATALDO, D.H. 2007. Fast response of freshwater consumers to a new trophic resource: predation on the recently introduced asian bivalve Limnoperna fortunei in the lower Paraná River, South America. Austral Ecol. 32(4):403-415.

The R Development Core Team. 2006. R: a language and environment for statistical computing. R Foundation for Statistical Computing, Vienna. http://www.r-project.org (último acesso em 11/03/2008).

WILLIAMSON, M. \& FITTER, A. 1996. The characters of successful invaders. Biol. Conserv. 78(1-2):163-170.

Recebido em 16/03/09

Versão reformulada recebida em 30/07/09

Publicado em 30/07/09 